\title{
Topical delivery of hexamidine
}

Nicola Parisi ${ }^{1}$, Miguel Paz-Alvarez ${ }^{1}$, Paul J. Matts ${ }^{2}$, Rebecca Lever ${ }^{1}$, Jonathan Hadgraft $^{1}$, Majella E. Lane ${ }^{1 *}$

${ }^{1}$ UCL School of Pharmacy

29-39 Brunswick Square

London

WC1N 1AX

United Kingdom

${ }^{2}$ Procter \& Gamble Technical Centres Ltd,

London Innovation Centre,

Whitehall Lane,

Egham, Surrey

TW20 9NW

United Kingdom

*Corresponding author

Email: majella.lane@btinternet.com

Tel: +44 2077535821

Fax: +44 8701659275 


\begin{abstract}
Hexamidine diisethionate (HEX D) has been used for its biocidal actions in topical preparations since the 1950s. Recent data also suggest that it plays a beneficial role in skin homeostasis. To date, the extent to which this compound penetrates the epidermis has not been reported nor how its topical delivery may be modulated. In the present work we set out to characterise the interaction of HEX D with the skin and to develop a range of simple formulations for topical targeting of the active. A further objective was to compare the skin penetration of HEX D with its corresponding dihydrochloride salt $(\mathrm{HEX} \mathrm{H})$ as the latter has more favourable physicochemical properties for skin uptake. Candidate vehicles were evaluated by in vitro Franz cell permeation studies using porcine skin. Initially, neat solvents were investigated and subsequently binary systems were examined. The solvents and chemical penetration enhancers investigated included glycerol, dimethyl isosorbide (DMI), isopropyl alcohol (IPA), 1,2-pentanol (1,2-PENT), polyethylene glycol (PEG) 200, propylene glycol (PG), propylene glycol monolaurate (PGML) and Transcutol ${ }^{\circledR} \mathrm{P}$ (TC). Of a total of 30 binary solvent systems evaluated only 10 delivered higher amounts of active into the skin compared with the neat solvents. In terms of topical efficacy, formulations containing PGML far surpassed all other solvents or binary combinations. More than $70 \%$ of HEX H was extracted from the skin following application in PG:PGML (50:50). Interestingly, the same vehicle effectively promoted skin penetration of HEX D but demonstrated significantly lower uptake into and through the skin (30\%). The findings confirm the unpredictable nature of excipients on delivery of actives with reference to skin even where there are minor differences in molecular structures. We also believe that they underline the ongoing necessity for fundamental studies on the interaction of topical excipients with the skin.
\end{abstract}

Key words: Hexamidine, skin, formulation, solvents, chemical penetration enhancers 


\section{Introduction}

Hexamidine diisethionate (HEX D) has been used as a biocide in topical preparations since 1950 (Robin, 1978) but recent evidence indicates it may have also have a beneficial effect on skin homeostasis. It has been suggested that the inhibitory action of HEX on inflammatory proteases in skin may result in modulation of skin ageing processes (Kimball et al., 2012). Synthesis of sphingolipid, fatty acid and cholesterol was downregulated in human skin equivalent cell cultures following incubation with HEX D, as was fatty acid and cholesterol uptake; the efflux of cholesterol was also upregulated (Osborne et al., 2009; Jarrold et al., 2010a). In vivo studies conducted with an emollient containing HEX D and other ingredients have also shown improved skin barrier function compared with controls (Jarrold et al., 2010b).

Surprisingly the disposition of HEX D in skin has not been examined to date. In a recent publication (Parisi et al., 2015) we reported the preparation of the dihydrochloride salt (HEX H) as well as the characterisation of both molecules. The rationale for preparation of HEX $\mathrm{H}$ was based on its likely more favourable properties for topical delivery compared with HEX D. Effective skin penetration has been observed for molecules with low molecular weights $(<500)$, low melting points and balanced lipophilic - hydrophilic properties (Hadgraft, 2004). Although HEX H was successfully prepared in the pure form, the melting point of the molecule was higher than that of HEX D. Thermal analysis indicated the melting points of HEX D and HEX $\mathrm{H}$ were $225^{\circ} \mathrm{C}$ and $266^{\circ} \mathrm{C}$ respectively. $\log \mathrm{D}$ values at $\mathrm{pH} 7.4$ were -0.74 for HEX $\mathrm{D}$ and -0.70 for HEX $\mathrm{H}$ respectively. The investigation of the physicochemical properties of HEX D and HEX $\mathrm{H}$ also included determination of UV absorption spectra, $\mathrm{pH}$ in aqueous solution, development of appropriate HPLC analytical methods as well as evaluation of solubility and stability in a broad range of solvents and selected binary solvent systems. Following on from these preformulation studies, the aim of the present work was to identify and develop simple formulations to deliver both HEX D and HEX $\mathrm{H}$ to the skin.

Because of the excellent barrier properties of the skin, the bioavailability of topically applied active ingredients is generally low. Chemical penetration enhancers (CPEs) are often included in pharmaceutical and cosmetic formulations to improve delivery of actives. Although the precise mechanism of action of most CPEs remains poorly understood, two major effects may be observed when they penetrate the 
stratum corneum (Hadgraft, 1999). Some CPEs, usually simple solvent type molecules, can alter the skin permeability by increasing the solubility of the permeant in the stratum corneum. Other enhancers may, instead, increase the diffusion coefficient of the permeant by intercalating into the highly organised lipid domain of the stratum corneum and disrupting its packing, thus making it more fluid (Lane, 2013). Finally, a synergistic effect may be obtained by combining in the same formulation chemical penetration enhancers having different mechanisms of action (Hadgraft, 1999).

Single solvent systems were selected as the starting point for the development and evaluation of optimal formulations for the topical delivery of HEX D and HEX H. Based on the solubility data for HEX D and HEX H in 19 solvents studied in our previous work (Parisi et al., 2015), propylene glycol (PG), glycerol and PEG 200 were identified as suitable candidate vehicles. Conducting in-vitro skin permeation studies using diffusion cells is by far the most accepted method used to investigate and screen a series of topical formulations in order to select those which perform better in terms of active ingredient release to the desired target (Franz, 1975; Organisation for Economic Cooperation and Development, 2004; Hirata et al., 2013). Permeation studies, however, only allow the quantification of the amount of active which cross the skin barrier. Therefore for this study they are coupled with mass balance studies which provide information on both the amount of active which remains on the skin surface and that which is delivered inside the skin (Tsai et al., 1992; OECD, 2004; Oliveira et al., 2012). A range of binary systems which combined the single solvents with other CPEs were subsequently prepared and examined. The choice of CPE was based on their miscibility with PG, glycerol and PEG 200 as well as reports in the literature detailing their applications in topical and transdermal delivery (Lane, 2013).

\section{Materials and Methods}

\subsection{Materials}

HEX D (Laboratoires Sérobiologiques, France) was a kind gift from Procter \& Gamble (U.K.), while HEX H was synthesized and purified in-house. Porcine ears were obtained from a local abattoir. Propylene glycol (PG), polyethylene glycol 200 (PEG 200) and HPLC grade isopropyl alcohol (IPA) were supplied by Fisher Scientific (U.K.). HPLC grade water, HPLC grade methanol and glycerol were 
provided by Sigma-Aldrich (U.K.). Dimethyl sulfoxide (DMSO) was supplied by VWR International (U.K.). LauroglycolTM 90 (Propylene glycol monolaurate, PGML) and Transcutol ${ }^{\circledR} \mathrm{P}$ (TC) were received as kind donations from Gattefossé (France). 1,2-pentanediol (1,2-PENT) was provided by Surfachem Group (U.K.). Dimethyl isosorbide Arlasolve ${ }^{\circledR}$ (DMI) was supplied by Croda International (U.K.). Phosphate buffered saline $(\mathrm{PBS})\left(\mathrm{pH}=7.3 \pm 0.2\right.$ at $\left.25^{\circ} \mathrm{C}\right)$ was prepared using Dulbecco A tablets (Oxoid, U.K.).

\subsection{Methods}

2.2.1 Preparation of HEX D and HEX $\mathrm{H}$ formulations and solubility determination 
HEX D and HEX H solutions $(0.1 \% \mathrm{w} / \mathrm{v})$ were prepared by placing $5 \mathrm{mg}$ of active ingredient in a screw top glass test tube containing a Teflon ${ }^{\circledR}$-coated magnetic stir bar. The concentration selected is the amount which is currently approved for use in personal care products (Cosmetic Ingredient Review Expert Panel, 2007). Solutions were allowed to stir and equilibrate overnight at $32 \pm 1{ }^{\circ} \mathrm{C}$. If no visible excess of active ingredient was found, the solution was used without further processing. If a saturated solution with a visible excess of drug was obtained, a sample was withdrawn and centrifuged at $13200 \mathrm{rpm}$ for $15 \mathrm{~min}$ at $32 \pm 1^{\circ} \mathrm{C}$ in an Eppendorf 5415R centrifuge (Eppendorf, Germany). The supernatant was then used without further processing. Finally, the concentrations of HEX D and HEX H in all solutions were determined by HPLC (Parisi et al., 2015). In vitro permeation and mass balance studies were performed with the following single solvent systems: $0.1 \%$ w/v HEX D in PG, glycerol and PEG 200 and 0.1\% w/v HEX H in PG, glycerol and PEG 200. In vitro permeation and mass balance studies were performed with the following binary solvent systems: 0.1\% w/v HEX D in PG:TC (50:50), glycerol:TC (50:50), PEG 200:TC (50:50), PG:DMI (50:50), glycerol:DMI (50:50), PG:1,2-PENT (50:50), glycerol:1,2-PENT (50:50), PEG 200:1,2-PENT (50:50), PG:IPA (50:50), glycerol:IPA (50:50), PEG 200:IPA (50:50) and HEX D at saturation solubility in PEG 200:DMI (50:50), PG:PGML (50:50), glycerol:PGML (50:50) and PEG 200:PGML (50:50). For HEX H, the same binary solvent systems as for HEX D were used, but the content of HEX H was $0.1 \%$ w/v. The solubility of HEX D and HEX $\mathrm{H}$ in the neat solvents was reported in our previous publication and for the binary systems solubility was determined in the same manner (Parisi et al., 2015). Briefly, an excess amount of active was added to each solvent in a glass test tube containing a Teflon ${ }^{\circledR}$-coated magnetic stir bar. The test tube was sealed with 
Parafilm $^{\circledR}$ and placed in a SUB 28 thermostatically controlled water bath (Grant Instruments, U.K.) equipped with a Telesystem HP 15 submersible magnetic stirrer (Variomag ${ }^{\circledR}$-USA, U.S.A.). The system was allowed to stir and equilibrate for $48 \mathrm{~h}$ at $32 \pm 1^{\circ} \mathrm{C}$ to obtain a saturated solution. After the $48 \mathrm{~h}$ period, a sample was withdrawn from the test tube and centrifuged at $13200 \mathrm{rpm}$ for $15 \mathrm{~min}$ at $32 \pm 1^{\circ} \mathrm{C}$ in an Eppendorf 5415R centrifuge (Eppendorf, Germany). Following sample dilution the concentration of the active was determined by HPLC.

\subsubsection{In vitro permeation studies}

All permeation studies were conducted using glass vertical Franz diffusion cells and porcine ear skin and followed procedures reported previously (Caon et al., 2010; Oliveira et al., 2012). Full thickness skin tissue was used for all experiments. The Franz cell experiments were conducted in a SUB 28 (Grant Instruments, U.K.) temperature controlled water bath equipped with a Telesystem HP 15 (Variomag ${ }^{\circledR}$ USA, U.S.A.) submersible magnetic stirrer. The skin temperature was equilibrated to $32 \pm 1^{\circ} \mathrm{C} .250 \mu \mathrm{L}$ of the test solution were then applied to the donor compartment which was not occluded. Samples of receptor solution were collected at 0, 6, 12, 20, 24, 28, 32, 45 and $48 \mathrm{~h}$. The sample volume was $100 \mu \mathrm{L}$ and, after removal an equal volume of fresh PBS at $32 \pm 1{ }^{\circ} \mathrm{C}$ was added to the receptor compartment. Finally, the HEX D and HEX H concentration in all the samples was determined by HPLC (Parisi et al., 2015).

\subsubsection{Mass balance studies and validation}

At the end of the $48 \mathrm{~h}$ permeation studies, the receptor solution was removed from the Franz cells. The donor solution was then withdrawn from each donor compartment and placed in a $10 \mathrm{~mL}$ volumetric flask. For hydrophilic solvents HPLC grade water was used for dilution and for hydrophobic solvents DMSO was used. After removing the donor solution, the skin surface was washed twice (each wash consisting of 5 solvent rinses) with HPLC grade water in the case of hydrophilic donor solutions and with DMSO for hydrophobic donor solutions. The Franz diffusion cells were then disassembled, the skin membranes were placed in 
centrifuge microtubes and extracted with DMSO:methanol (50:50) on a rotor for 24 $\mathrm{h}$ at $32 \pm 1{ }^{\circ} \mathrm{C}$. Two cycles of sonication $(15 \mathrm{~min})$ and centrifugation at $13200 \mathrm{rpm}$ (15 min) were performed in order to complete the extraction. The HEX D and HEX $\mathrm{H}$ concentrations in all the diluted donor solutions, washing samples and extraction samples were determined by HPLC (Parisi et al., 2015). The total active ingredient recovery (\%) was then used as an indicator of the reliability of the procedure and values within the range from $80 \%$ to $120 \%$ were considered acceptable (OECD, 2011)

The mass balance methods were validated using a $0.1 \% \mathrm{w} / \mathrm{v}$ solution of HEX D in PG for hydrophilic vehicles and a $0.1 \%$ w/v solution of HEX H in PG:PGML for hydrophobic vehicles. The Franz diffusion cells were assembled as for typical permeation studies but receptor compartments contained no PBS. Following equilibration to the experimental temperature $\left(32 \pm 1^{\circ} \mathrm{C}\right), 250 \mu \mathrm{L}$ of donor solution were applied to the skin surface for $6 \mathrm{~h}$. At the end of the $6 \mathrm{~h}$ period, the PG donor solution was withdrawn and placed in a $10 \mathrm{~mL}$ volumetric flask and diluted with HPLC grade water. The solution of HEX H in PG:PGML was diluted with DMSO. After removing the donor solution, the skin surface was washed 5 times with HPLC grade water for the PG donor solution, and with DMSO for the PG:PGML solution. The Franz diffusion cells were then disassembled, the skin membranes were placed in centrifuge microtubes and extracted as described for the mass balance studies. HEX D and HEX H concentrations in all the diluted donor solution, washing samples and extraction samples were determined by HPLC (Parisi et al., 2015); validation required total recovery values of between $95 \%$ and $105 \%$.

\subsubsection{Data treatment and statistical analysis}

The data analysis was performed using Microsoft ${ }^{\circledR}$ Excel 2010 (Microsoft Corporation, U.S.A.) and OriginPro ${ }^{\circledR}$ 9.1.0 (OriginLab Corporation, U.S.A.). The data points falling between the LOQ and the LOD were not excluded and were used in the same manner as those above the LOQ (Keizer et al., 2015). The data below the LOD were instead excluded. As a result, when all the observations constituting a sample were below the LOD, this was reported as not detected (ND). However, when the observations constituting a sample were both above and below the LOD, the latter were statistically estimated in order to be able to calculate meaningful descriptive statistics, such as the sample arithmetic mean, standard deviation (SD) 
and standard error of the mean (SEM). The results are presented as mean $\pm \mathrm{SD}$ or mean \pm SEM. The normality of the data was assessed using the Shapiro-Wilk and Kolmogorov-Smirnov tests. Parametric statistical tests (one-way ANOVA and independent-samples t-test) were used to compare means and investigate statistical differences for normally distributed data. When the data showed a non-normal distribution, the Kruskal-Wallis test and the Mann-Whitney u-test (non-parametric tests) were used as alternatives for the one-way ANOVA and independent-samples ttest, respectively. The one-sample t-test was used to compare the mean of a normal sample with a ND sample whose mean was considered to be zero. Finally, a probability of $p<0.05$ was considered statistically significant.

\section{Results and Discussion}

\subsection{Mass balance method validation}

The results of the validation of the mass balance methods for the solutions of HEX D in PG and HEX H in PG:PGML are shown in Figure 1. 


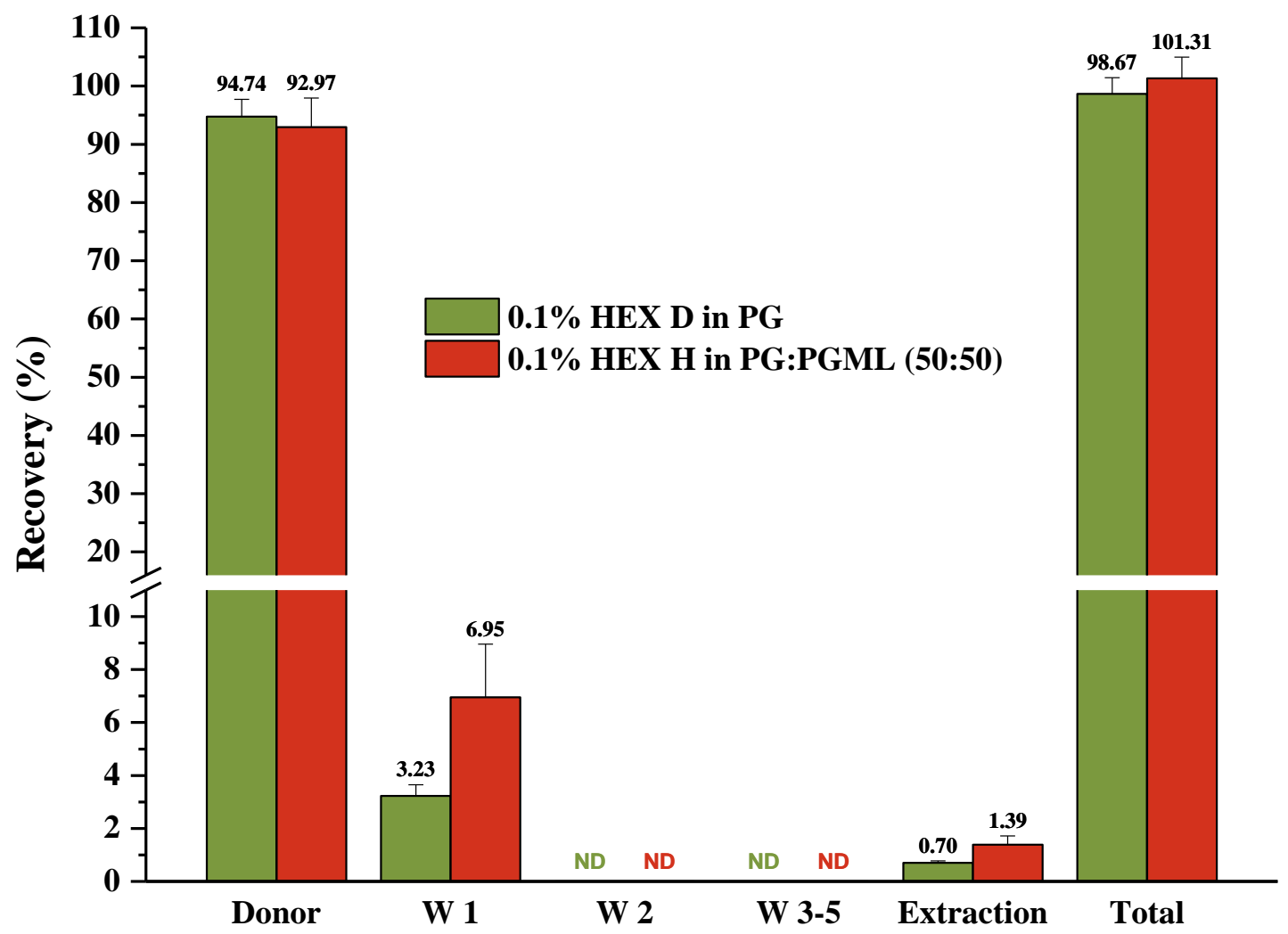

Figure 1 Residual donor solution, washing (W), extraction and total recovery of HEX D and HEX $\mathrm{H}$ for HEX D in PG and HEX H in PG:PGML $(4 \leq n \leq 5$; mean \pm SD)

HEX D and HEX $H$ were largely recovered from the skin surface in the residual donor solutions plus washings (Figure 1). The recovery of the active ingredients from the residual donor solutions accounted for more than $90 \%$ of the applied doses, while the first washing consisted of between $3 \%$ and $7 \%$ of the dose. No HEX D and HEX H were detected in washing steps 3, 4 and 5 for the solution of HEX D in PG and the solution of HEX $\mathrm{H}$ in PG:PGML. As a result, only two washing steps were performed in all subsequent mass balance studies. Following skin extraction, small quantities of active were recovered for the solutions of HEX D in PG and HEX $\mathrm{H}$ in PG:PGML $(0.70 \%$ and $1.39 \%$ of the applied doses, respectively). The efficiency of the mass balance methods was validated by the total recovery values which were within the range of $95 \%$ to $105 \%$. 


\subsection{In vitro permeation and mass balance studies using single solvent systems}

No permeation was observed for solutions of HEX D in PG, glycerol and PEG 200. The results of the mass balance studies are presented in Table 1. More than 90\% of the applied HEX D was recovered from the skin surface. In addition, the skin surface recovery for each vehicle was not statistically different from the respective total recovery (one-way ANOVA, $p>0.05$ ) which ranged from $91.36 \%$ for the PEG 200 solution to $93.10 \%$ for the glycerol solution. Values for skin surface and total recovery were statistically higher than for skin extraction (one-way ANOVA, $p<$ 0.001). Less than $1 \%$ of the applied HEX D was delivered into the skin. This is not surprising considering that the molecular weight, the $\log \mathrm{D}_{\mathrm{o} / \mathrm{w}}$ at $\mathrm{pH}=7.4$ and the melting point of HEX D are not ideal for topical drug delivery (Parisi et al., 2015). In addition, although PG, glycerol and PEG 200 were specifically selected because of the high solubility of HEX D in these solvents, this may have limited HEX D distribution into the stratum corneum. No significant differences were found between individual solvents for values of active extracted from skin (one-way ANOVA, $p>$ $0.05)$. 
Table 1 Skin surface, skin extraction and total HEX D recovery (\%) for single and binary solvent systems following application to porcine skin for $48 \mathrm{~h}$. The amounts $(\mu \mathrm{g})$ of HEX D extracted from the skin are also shown in brackets. $(4 \leq \mathrm{n} \leq 5$; mean \pm SD)

\begin{tabular}{|l|l|l|l|}
\hline Vehicle & Skin surface & Skin extraction & Total recovery \\
\hline PG & $91.95 \pm 6.16$ & $0.72 \pm 0.09(1.77 \pm 0.22)$ & $92.66 \pm 6.13$ \\
\hline Glycerol & $92.23 \pm 11.99$ & $0.87 \pm 0.18(1.95 \pm 0.41)$ & $93.10 \pm 11.97$ \\
\hline PEG 200 & $90.38 \pm 1.48$ & $0.99 \pm 0.28(2.48 \pm 0.70)$ & $91.36 \pm 1.21$ \\
\hline PG:TC & $89.95 \pm 2.10$ & $0.46 \pm 0.02(1.20 \pm 0.05) \dagger$ & $90.40 \pm 2.10$ \\
\hline Glycerol:TC & $86.62 \pm 1.25$ & $0.46 \pm 0.06(1.30 \pm 0.16) \dagger$ & $87.08 \pm 1.26$ \\
\hline PEG 200:TC & $88.86 \pm 1.52$ & $0.64 \pm 0.05(1.63 \pm 0.13)$ & $89.49 \pm 1.48$ \\
\hline PG:DMI & $106.97 \pm 2.00$ & ND $\dagger$ & $106.97 \pm 2.00$ \\
\hline Glycerol:DMI & $100.21 \pm 5.25$ & ND $\dagger$ & $100.21 \pm 5.25$ \\
\hline PEG 200:DMI & $90.94 \pm 0.74$ & $1.03 \pm 0.41(1.42 \pm 0.57) \dagger$ & $91.97 \pm 0.73$ \\
\hline PG:1,2-PENT & $89.11 \pm 5.09$ & $1.01 \pm 0.16(2.38 \pm 0.37) *$ & $90.13 \pm 4.96$ \\
\hline Glycerol:1,2-PENT & $93.69 \pm 6.30$ & $1.04 \pm 0.18(2.42 \pm 0.41)$ & $94.73 \pm 6.26$ \\
\hline PEG 200:1,2-PENT & $85.77 \pm 5.08$ & $0.99 \pm 0.18(2.37 \pm 0.44)$ & $86.76 \pm 5.07$ \\
\hline PG:IPA & $87.07 \pm 5.13$ & $3.48 \pm 1.69(8.48 \pm 4.14)^{*}$ & $90.55 \pm 3.68$ \\
\hline Glycerol:IPA & $86.20 \pm 1.10$ & $2.98 \pm 0.96(7.37 \pm 2.37)^{*}$ & $89.18 \pm 2.02$ \\
\hline PEG 200:IPA & $88.50 \pm 4.71$ & $1.70 \pm 0.59(4.23 \pm 1.48)^{*}$ & $90.19 \pm 4.60$ \\
\hline
\end{tabular}

Similarly for HEX $\mathrm{H}$, no permeation was observed after the $48 \mathrm{~h}$ in-vitro permeation studies for PG, glycerol and PEG 200 solutions (Table 2). 
Table 2 Skin surface, skin extraction and total HEX H recovery (\%) for single and binary solvent systems following application to porcine skin for $48 \mathrm{~h}$. The amounts $(\mu \mathrm{g})$ of HEX H extracted from the skin are also shown in brackets. $(3 \leq n \leq 5$; mean \pm SD)

\begin{tabular}{|l|l|l|l|}
\hline Vehicle & Skin surface & Skin extraction & Total recovery \\
\hline PG & $92.77 \pm 4.23$ & $1.51 \pm 0.91(3.63 \pm 2.18)$ & $94.29 \pm 3.36$ \\
\hline Glycerol & $99.10 \pm 11.62$ & $1.03 \pm 0.25(2.39 \pm 0.57)$ & $100.13 \pm 11.49$ \\
\hline PEG 200 & $86.04 \pm 1.67$ & $2.18 \pm 0.62(4.48 \pm 1.27)$ & $88.23 \pm 1.53$ \\
\hline PG:TC & $94.24 \pm 2.73$ & $0.71 \pm 0.10(1.26 \pm 0.19) \dagger$ & $94.95 \pm 2.82$ \\
\hline Glycerol:TC & $98.46 \pm 5.50$ & $0.46 \pm 0.15(0.94 \pm 0.31 \dagger$ & $98.92 \pm 5.40$ \\
\hline PEG 200:TC & $97.62 \pm 2.21$ & $0.57 \pm 0.03(1.20 \pm 0.07) \dagger$ & $98.19 \pm 2.19$ \\
\hline PG:DMI & $94.53 \pm 2.03$ & $0.53 \pm 0.21(1.36 \pm 0.55) \dagger$ & $95.06 \pm 2.20$ \\
\hline Glycerol:DMI & $92.79 \pm 4.02$ & $0.40 \pm 0.27(1.02 \pm 0.69) \dagger$ & $93.20 \pm 4.22$ \\
\hline PEG 200:DMI & $96.13 \pm 1.97$ & $0.85 \pm 0.04(2.01 \pm 0.09)$ & $96.98 \pm 1.99$ \\
\hline PG:1,2-PENT & $91.17 \pm 3.82$ & $1.04 \pm 0.24(2.07 \pm 0.47) \dagger$ & $92.21 \pm 3.81$ \\
\hline Glycerol:1,2-PENT & $94.27 \pm 3.92$ & $0.79 \pm 0.08(1.78 \pm 0.18)$ & $95.06 \pm 3.91$ \\
\hline PEG 200:1,2-PENT & $95.89 \pm 2.47$ & $1.14 \pm 0.25(2.30 \pm 0.50) \dagger$ & $97.03 \pm 2.23$ \\
\hline PG:IPA & $97.52 \pm 4.34$ & $1.12 \pm 0.13(2.45 \pm 0.28)$ & $98.65 \pm 4.29$ \\
\hline Glycerol:IPA & $101.00 \pm 4.20$ & $1.46 \pm 0.29(3.08 \pm 0.60)$ & $102.47 \pm 4.40$ \\
\hline PEG 200:IPA & $102.62 \pm 3.34$ & $1.37 \pm 0.18(2.96 \pm 0.39) \dagger$ & $103.98 \pm 3.44$ \\
\hline$\dagger$ Binary solvent system which delivered significantly lower amount of active compared with neat solvent \\
\hline
\end{tabular}

More than $85 \%$ of the applied HEX $\mathrm{H}$ remained on the skin surface, while the skin extraction recoveries ranged from $1.03 \%$ for glycerol solution to $2.18 \%$ for PEG 200. Values for skin surface and total recovery were not significantly different (oneway ANOVA, $p>0.05$ ); but were statistically higher than the respective skin extraction recovery values (one-way ANOVA, $p<0.001$ ). No statistical differences were found (one-way ANOVA, $p>0.05$ ) between amounts of active extracted from the skin for the individual formulations. The conversion of HEX D to HEX H does appear to impact on topical delivery, subject to the vehicle selected. Statistically higher amounts of HEX H were extracted from the skin for the PG and PEG 200 vehicles compared with the corresponding HEX D solutions (Mann-Whitney u-test, $p$ $<0.01$; independent-samples t-test, $p<0.05)$. However, no significant differences were found between the amounts of the two actives extracted from the skin for the glycerol vehicle (independent-samples t-test, $p>0.05$ ). 


\subsection{In-vitro permeation and mass balance studies using binary solvent systems}

\subsubsection{Binary systems of PG, glycerol and PEG 200 with TC}

Binary solvent systems consisting of PG, glycerol and PEG 200 in combination with TC did not promote HEX D delivery through the skin. No active was found in the receptor compartment following the in-vitro permeation studies with solutions of HEX D in PG:TC, glycerol:TC and PEG 200:TC (Table 1). Less than $0.65 \%$ of the applied HEX D was delivered into skin by binary combinations of PG, glycerol or PEG 200 with TC (Table 1). Unsurprisingly, there were no significant differences between the skin surface and the total recoveries (one-way ANOVA, $p>0.05$ ) which are significantly higher than the corresponding skin extraction recoveries (one-way ANOVA, $p<0.001)$. Although the overall topical delivery of HEX D was poor, the amount of active extracted from the skin for PEG 200:TC was significantly higher than values for PG:TC and glycerol:TC (one-way ANOVA, $p<0.01$ ).

As for HEX D most of the HEX H remained on the skin surface (Table 2) and corresponding recovery values are not statistically different from those for total recovery (one-way ANOVA, $p>0.05$ ). The amount of HEX $\mathrm{H}$ extracted from skin was less than $0.75 \%$ of the applied dose for all solutions and significantly lower than the respective skin surface and total recoveries (one-way ANOVA, $p<0.001$ ). There are no statistical differences in amounts of HEX H delivered into skin for the binary systems (one-way ANOVA, $p>0.05$ ). In contrast to what was observed for the single solvent systems, the use of the dihydrochloride salt in place of the diisethionate did not improve the topical delivery of HEX. The amounts of HEX H extracted from the skin for glycerol:TC and PEG 200:TC were, instead, significantly lower than amounts of HEX D extracted from skin for the same vehicles (independent-samples t-test, $p<0.05$ and $p<0.001$, respectively). No differences were found between amounts of HEX $\mathrm{H}$ and the HEX D extracted for PG:TC (independent-samples t-test, $p>0.05$ ). A possible explanation for this is that HEX H is 5.4-fold more soluble in TC than HEX D (2.00 and $0.37 \mathrm{mg} / \mathrm{mL}$ respectively). Therefore, the higher solubility should result in a lower thermodynamic activity of HEX $\mathrm{H}$ in the binary solvent systems compared with HEX D. Ultimately this would result in a reduced driving force for HEX $\mathrm{H}$ into the skin despite the more favourable physicochemical properties of HEX H for topical delivery compared with HEX D. 
We hypothesised that the use of TC in combination with PG, glycerol and PEG 200 might enhance the topical delivery of HEX D and HEX $\mathrm{H}$ in comparison to delivery in single solvents. Harrison et al. (1996) and Puglia and Bonina (2008) reported increased fluxes across human skin for 4-cyanophenol and atenolol, respectively, and suggested that this reflected the ability of TC to facilitate solubilisation of active in the skin. However, the single solvent systems of HEX D and HEX $\mathrm{H}$ clearly performed better than the binary solvent systems. PG delivered significantly higher amounts of HEX D or HEX $\mathrm{H}$ into the skin compared with the corresponding solutions in PG:TC (Mann-Whitney u-test, $p<0.01$ and $p<0.05$, respectively). A similar trend was observed for solutions of HEX D and HEX H in glycerol (independent-samples t-test, $p<0.05$ and $p<0.01$, respectively) and for HEX H in PEG 200 (independent-samples t-test, $p<0.05$ ). Finally, the amount of HEX D delivered from PEG 200 was not statistically different from that delivered by the PEG 200:TC vehicle (independent-samples t-test, $p=0.052$ ). The excellent solubilising properties and the safety of TC are two of the main reasons behind its long-standing use in pharmaceutical and cosmetic products (Sullivan Jr. et al., 2014). $\mathrm{TC}$ is also widely used for its enhancing action on the transdermal delivery of active ingredients and for its ability to promote topical delivery via formation of intracutaneous depots (Osborne, 2011). This last effect was particularly appealing considering that the aim of this work was the topical delivery of HEX D and HEX H. However, as noted by Osborne (2011) it is difficult to make predictions of the efficacy of $\mathrm{TC}$ in enhancing the permeation of active ingredients. The precise mechanism of action of this compound where it does function as a CPE is still not fully understood. We have also recently reported the superior penetration of TC compared with PG and butylene glycol in human skin in vitro where saturated solutions of a different model active were investigated (Hadgraft and Lane, 2016).

\subsubsection{Binary systems of PG, glycerol and PEG 200 with DMI}

Permeation was not observed for HEX D in PG:DMI, glycerol:DMI and the saturated solution of HEX D in PEG 200:DMI. No HEX D was detected inside the skin for PG:DMI and glycerol:DMI, while 1\% of the applied dose was delivered from the saturated solution of HEX D in PEG 200:DMI (Table 1). The measured saturated solubility of HEX D in this vehicle at $32^{\circ} \mathrm{C}$ was $0.55 \pm 0.04 \mathrm{mg} / \mathrm{mL}(\mathrm{n}=3)$. Thus the amount in the skin corresponded to $1.42 \pm 0.57 \mu \mathrm{g}$ of HEX D, a statistically 
higher amount than the PG:DMI and glycerol:DMI vehicles (one-sample t-test, $p<$ 0.01). Skin surface recovery of HEX D from the saturated PEG 200:DMI solution was not significantly different from the total recovery (one-way ANOVA, $p>0.05$ ). As for HEX D, no permeation was observed for solutions of HEX H in PG:DMI, glycerol:DMI and PEG 200:DMI (Table 2).

In contrast to HEX D, binary solvent systems of DMI with PG, glycerol and PEG 200 delivered HEX H into the skin although the amounts were low, with less than $0.90 \%$ of the applied amount recovered (Table 2). The PG:DMI and the PEG 200:DMI systems delivered statistically similar amounts of HEX H into the skin (independent-samples t-test, $p>0.05$ ). However, while no significant difference was found between the former and the glycerol:DMI vehicle (independent-samples t-test, $p>0.05)$, the latter delivered a significantly higher amount of HEX H into the skin than the glycerol:DMI (50:50) solution (independent-samples t-test, $p<0.01$ ). Although the DMI systems were not particularly effective in targeting HEX D and HEX $\mathrm{H}$ to the skin, differences were observed in the overall topical delivery of the two salts. Significantly higher amounts of HEX H were delivered into the skin from the PG:DMI and glycerol:DMI solutions (one-sample t-test, $p<0.01$ and $p<0.05$, respectively). However, no differences were found between the solution of HEX $\mathrm{H}$ in PEG 200:DMI and the saturated solution of HEX D in the same system (independent-samples t-test, $p=0.051$ ). This last outcome was particularly interesting considering that HEX D had maximal thermodynamic activity in PEG 200:DMI. It may be concluded that for this set of binary systems at least, the use of the dihydrochloride rather than the diisethionate salt is preferred for optimum delivery into the skin.

The amounts of HEX D and HEX $\mathrm{H}$ extracted from the skin for the PG solutions were significantly higher than those for the corresponding PG:DMI (50:50) solutions (one-sample t-test, $p<0.001$ and Mann-Whitney u-test, $p<0.01$, respectively). The same was observed for the glycerol solutions which, again, delivered more of each active into the skin than the glycerol:DMI vehicle (onesample t-test, $p<0.001$ and independent-samples t-test, $p<0.05$, respectively). The topical delivery of HEX D from the PEG 200 solution was significantly higher than for the saturated solution of HEX D in PEG 200:DMI (independent-samples t-test, $p$ $<0.05)$, no differences were found between the same solvent systems containing HEX H (independent-samples t-test, $p>0.05$ ).Contrasting opinions on the efficacy 
of DMI as a CPE are reported and, in some studies, DMI has been used as a control or diluent for other penetration enhancers because it was considered to have no enhancing action (Bennett et al., 1985; Funke et al., 2002).

\subsubsection{Binary systems of PG, glycerol and PEG 200 with 1,2-PENT}

No HEX D penetrated through the skin from the PG:1,2-PENT, glycerol:1,2PENT and PEG 200:1,2-PENT vehicles (Table 1). All three systems delivered 1\% of the applied HEX D dose into the skin. As a result, the skin extraction recoveries were statistically lower than the corresponding skin surface and total recoveries (oneway ANOVA, $p<0.001)$. Amounts of HEX D recovered from the skin surface, are not significantly different from values for total recovery (one-way ANOVA, $p>$ 0.05). The three binary solvent systems delivered similar amounts of HEX D into skin (one-way ANOVA, $p>0.05$ ).

As for HEX D, no HEX H permeated from the PG:1,2-PENT, glycerol:1,2PENT and PEG 200:1,2-PENT solutions (Table 2). The majority of the HEX H was also found on the skin surface with most values $>90 \%$ of the applied dose and these were not significantly different from the corresponding values for total recovery (one-way ANOVA, $p>0.05$ ). The percentage of the applied HEX $\mathrm{H}$ recovered inside the skin ranged from $0.79 \%$ for the glycerol:1,2-PENT system to $1.14 \%$ for the PEG 200:1,2-PENT. As a result, all the extraction recoveries were statistically lower than the corresponding skin surface and total recoveries (one-way ANOVA, $p$ $<0.001)$. Interestingly, as already seen for HEX D, no significant differences were observed between the amounts of HEX $\mathrm{H}$ that the three vehicles delivered into the skin (one-way ANOVA, $p>0.05$ ).

Overall, the topical delivery of HEX from the 1,2-PENT systems was not significantly affected by the choice of salt. The amounts of active extracted from the skin for the PG:1,2-PENT and PEG 200:1,2-PENT formulations of HEX D were not significantly different from those for the corresponding HEX $\mathrm{H}$ solutions (MannWhitney u-test, $p>0.05$ and independent-samples t-test, $p>0.05$, respectively). In contrast, the glycerol:1,2-PENT solution delivered a statistically higher amount of HEX D into the skin than HEX H (independent-samples t-test, $p<0.05$ ). The use of 1,2-PENT as a CPE is relatively recent. There is little published data on the penetration enhancement properties of this solvent. Duracher et al. (2009) demonstrated the efficacy of 1,2-PENT for enhancement of delivery of the model 
hydrophilic active, caffeine, in porcine ear skin, under finite dose conditions. The authors not only showed that 1,2-PENT was more efficient than PG, ethanol and water but also observed a concentration dependent effect of 1,2-PENT. In the present study the combination of 1,2-PENT with PG, glycerol and PEG 200 did not improve the topical delivery of HEX D and HEX $\mathrm{H}$ beyond that achieved with the single solvent systems. No significant differences were detected between the amounts of HEX D and HEX H delivered into the skin by neat glycerol and the corresponding 1,2-PENT binary systems (independent-samples t-test, $p>0.05$ ). For PEG 200, no statistical differences were found between HEX D delivery from the neat solvent and in PEG 200:1,2-PENT (independent-samples t-test, $p>0.05$ ). Similarly, PEG 200 was more efficient than PEG 200:1,2-PENT in delivering HEX H (independentsamples t-test, $p<0.05)$. In contrast, the PG:1,2-PENT vehicle did deliver a higher amount of HEX D into the skin than neat PG (independent-samples t-test, $p<0.05$ ), however the opposite effect was observed for HEX H (Mann-Whitney u-test, $p<$ $0.05)$.

\subsubsection{Binary systems of PG, glycerol and PEG 200 with IPA}

No HEX D permeated from the PG:IPA, glycerol:IPA and PEG 200:IPA solutions (Table 1). Most of the applied HEX D was recovered from the skin surface (Table1). With the exception of the glycerol:IPA system, where skin surface and total recoveries were statistically different (one-way ANOVA, $p<0.05$ ), no significant differences were found between the values for skin surface recovery and the corresponding values for total recovery of the other IPA binary solvent systems (one-way ANOVA, $p>0.05$ ). All solutions delivered $>1.5 \%$ of the applied HEX D dose into the skin. The amount of HEX D recovered inside the skin ranged from 4.23 $\pm 1.48 \mu \mathrm{g}$ for PEG 200:IPA to $8.48 \pm 4.14 \mu \mathrm{g}$ for PG:IPA but no significant differences were observed between the vehicles (one-way ANOVA, $p>0.05$ ).

No HEX H was found in the Franz cell receptor compartments for the PG:IPA, glycerol:IPA and PEG 200:IPA solutions (Table 2). More than 95\% of the active was recovered from the skin surface while less than $1.5 \%$ was found inside the membrane. The skin extraction recoveries were thus significantly lower than the corresponding skin surface and total recoveries (one-way ANOVA, $p<0.001$ ) 
which, in turn, did not show statistical differences (one-way ANOVA, $p>0.05$ ). All binary solvent systems delivered similar amounts of HEX $\mathrm{H}$ into the skin and no statistical differences were observed between them (one-way ANOVA, $p>0.05$ ).

Interestingly, the use of IPA in combination with PG or glycerol facilitated the topical delivery of hexamidine as the diisethionate rather than as the dihydrochloride salt. The PG:IPA and glycerol:IPA vehicles delivered significantly higher amounts of HEX D into the skin than the corresponding solutions of HEX $\mathrm{H}$ (independentsamples t-test, $p<0.05)$. No statistical differences were found between the amounts of HEX D and HEX H extracted from the skin for the PEG 200:IPA solutions (independent-samples t-test, $p>0.05$ ). The addition of IPA to PG, glycerol or PEG 200 was also effective in enhancing the topical delivery of HEX D compared with the effects of the neat vehicles, while no effect was observed for HEX H. The PG:IPA, glycerol:IPA and PEG 200:IPA vehicles delivered statistically higher amounts of HEX D into the skin than PG, glycerol and PEG 200 alone (independentsamples t-test, $p<0.05, p<0.01$ and $p<0.05$, respectively). In contrast, no significant differences were found between the amounts of HEX $\mathrm{H}$ extracted from the skin for the neat PG and glycerol solutions and the related binary systems with IPA (independent-samples t-test, $p>0.05$ ). In addition, the topical delivery of HEX $\mathrm{H}$ from PEG 200 was significantly higher than that from PEG 200 in combination with IPA (independent-samples t-test, $p<0.05$ ). IPA is widely used in dermal and transdermal products because of its established penetration enhancing activity (Lane, 2013) but clearly it is ineffective here when combined with PEG 200 for HEX H.

\subsubsection{Binary systems of PG, glycerol and PEG 200 with PGML}

The respective values for saturation solubility of $\mathrm{HEX} \mathrm{D}$ at $32^{\circ} \mathrm{C}$ in the PG:PGML, glycerol:PGML and PEG 200:PGML vehicles were determined to be $0.44 \pm 0.02,0.46 \pm 0.03$ and $0.36 \pm 0.02 \mathrm{mg} / \mathrm{mL}(\mathrm{n}=3)$. The permeation profile of HEX D from a saturated solution in PG:PGML is shown in Figure 2. No permeation was observed for the saturated solutions of HEX D in glycerol:PGML and PEG 200:PGML. 


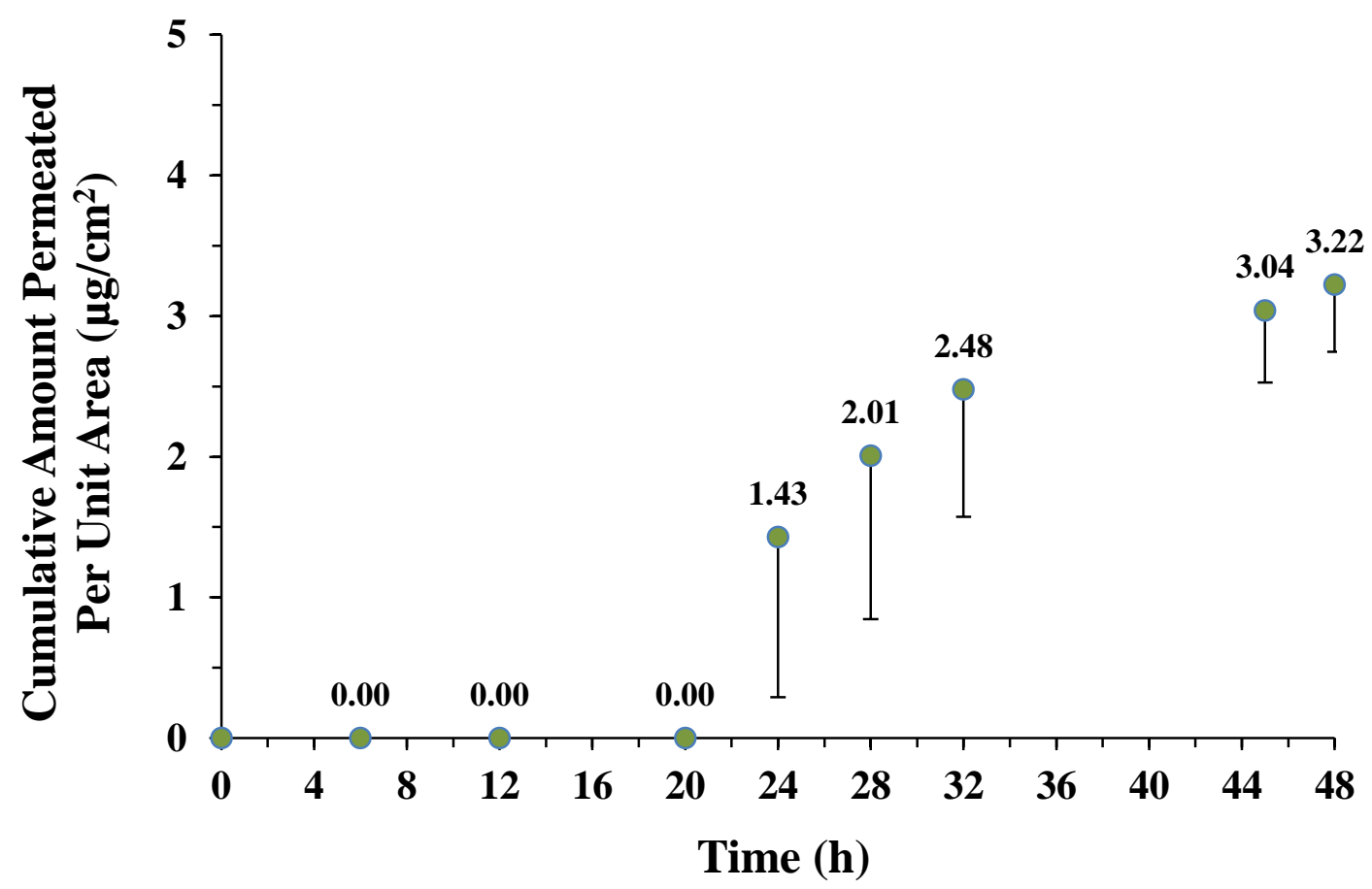

Figure 2 Cumulative amount of HEX D permeated over $48 \mathrm{~h}$ from a saturated solution in PG:PGML $(\mathrm{n}=5 ;$ mean $\pm \mathrm{SD})$

HEX D was detected in the Franz cell receptor compartment $24 \mathrm{~h}$ after the application of a saturated solution in PG:PGML. Although initially the active permeated at a constant rate, the flux decreased after $32 \mathrm{~h}$ and the permeation profile reached a plateau. Mass balance studies indicated that $34.06 \%$ of the applied HEX D dose was found on the skin surface (Figure 3). It is possible that the decrease of HEX D flux reflects depletion of the active itself from the donor solution. The low plateau value may also indicate stranding of the active or crystallization in skin (Hadgraft and Lane, 2016). Finally, despite the high variability observed, the amounts of HEX D permeated were statistically different from zero at all time points (one-sample ttest, $p<0.05)$. 


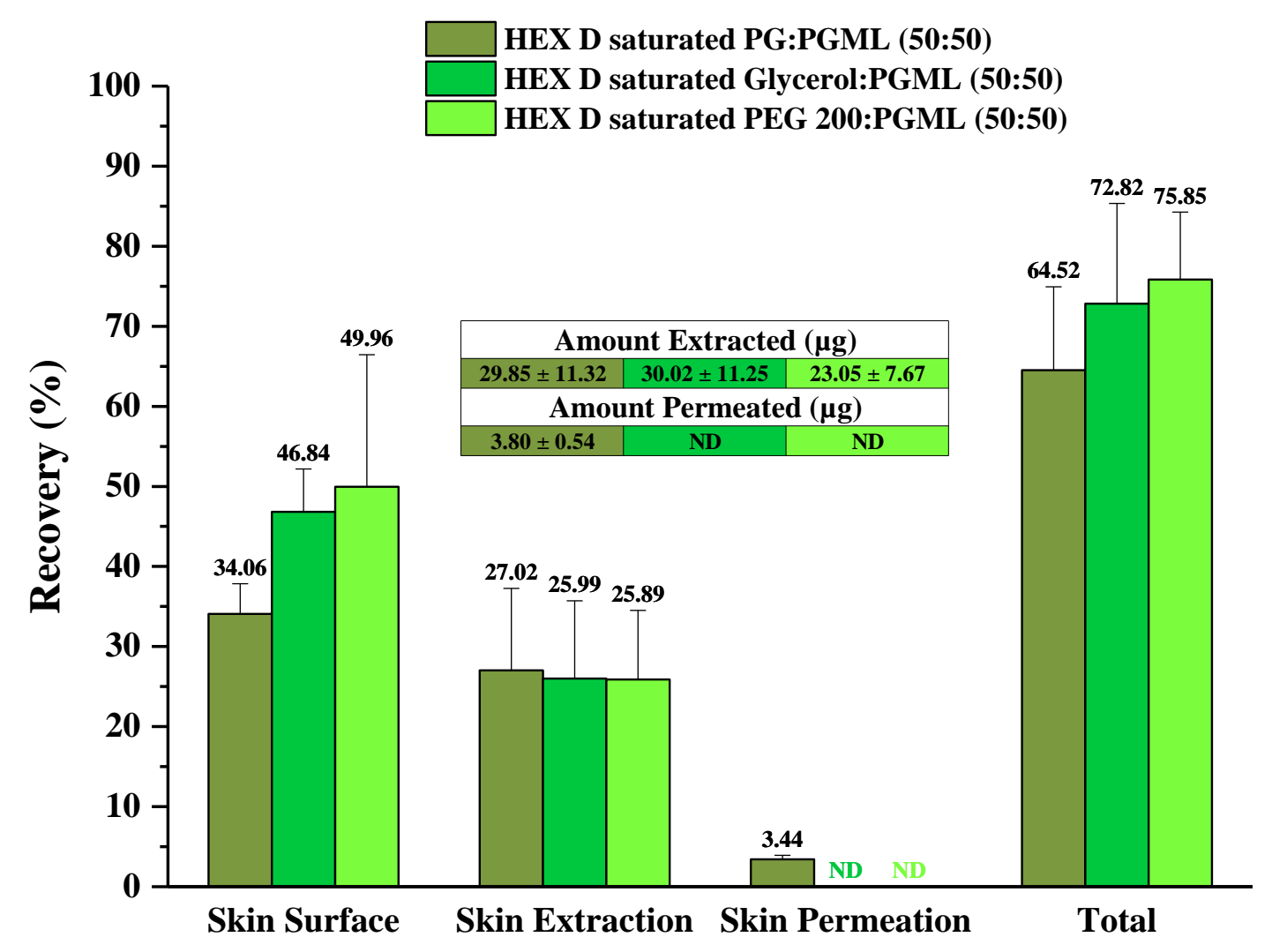

Figure 3 Percentage of HEX D on skin surface, extracted from skin, permeation at $48 \mathrm{~h}$ and total recovery for saturated PG:PGML, glycerol:PGML and PEG 200:PGML solutions.

Actual amounts of active recovered by extraction and measured in receptor compartment also

$$
\text { inset }(\mathrm{n}=5 \text {; mean } \pm \mathrm{SD})
$$

Figure 3 shows that all the binary solvent systems delivered just over $25 \%$ of the applied HEX D into the skin. For the PG:PGML system, the skin surface and the skin extraction recovery values were not statistically different (one-way ANOVA, $p$ $>0.05$ ) but were both significantly higher than the amounts which permeated through the skin (one-way ANOVA, $p<0.01$ ). The results for the glycerol:PGML and the PEG 200:PGML systems contrast with those for PG:PGML, primarily because the respective skin surface recoveries were significantly higher than the skin extraction values (one-way ANOVA, $p<0.05$ ). Nonetheless, the values are still significantly lower than corresponding total amounts recovered (one-way ANOVA, $p$ $<0.01$ and $p<0.05$, respectively). The topical delivery of HEX D ranged from 23.05 $\pm 7.67 \mu \mathrm{g}$ for PEG 200:PGML to $30.02 \pm 11.25 \mu \mathrm{g}$ for glycerol:PGML, however, no statistical differences were observed between the three binary solvent systems (oneway ANOVA, $p>0.05)$. Finally, the total recovery values for all vehicles were below the accepted range of $80-120 \%$ (OECD, 2011).. As HEX D was previously 
found to be stable in both PG:PGML and PBS (Parisi et al., 2015), the low total recovery might be attributed to interaction and/or degradation by skin enzymes.

For HEX H, no permeation was observed for PG:PGML, glycerol:PGML and PEG 200:PGML. Results for the mass balance evaluation are shown in Figure 4.

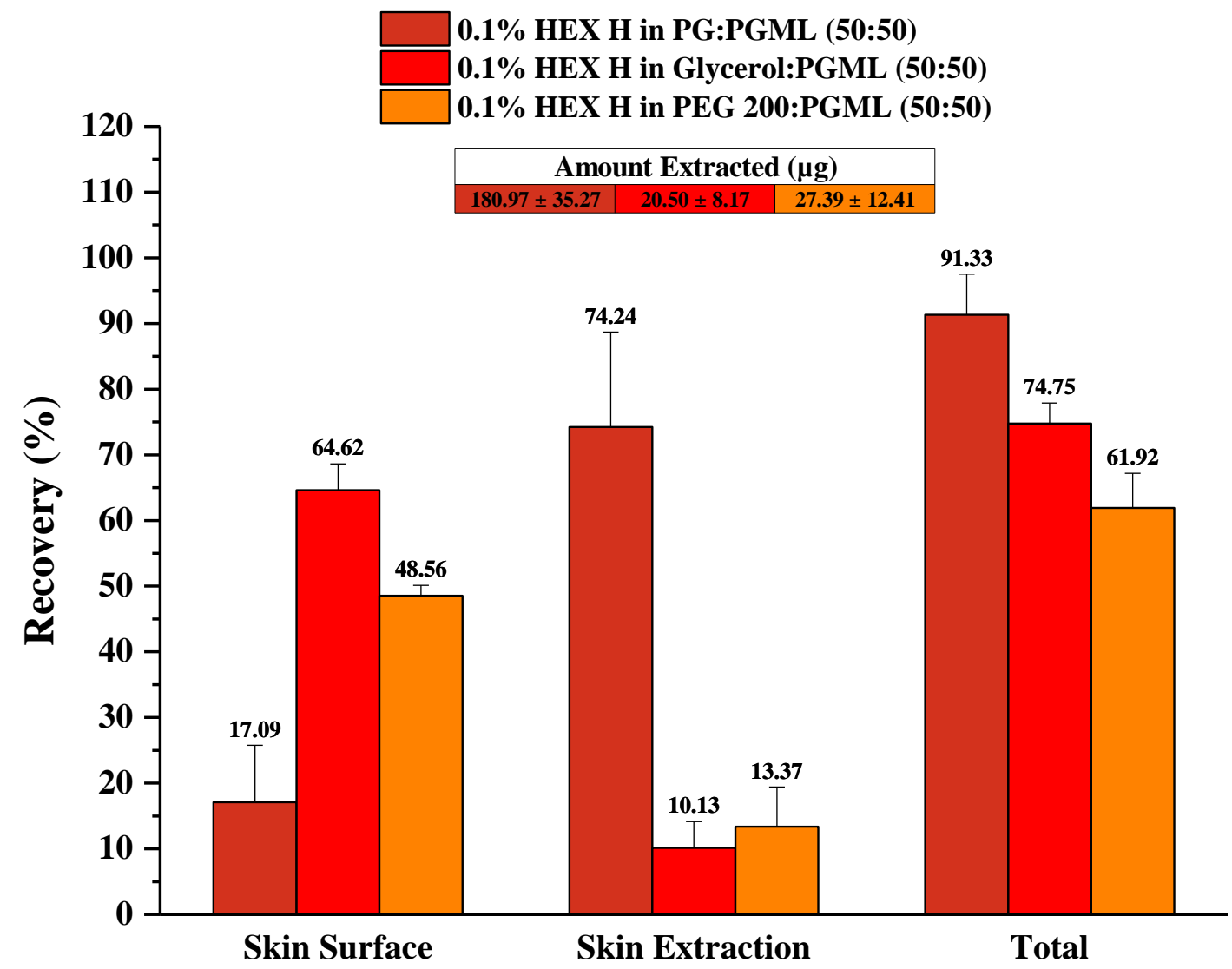

Figure 4 Percentage of HEX H recovered from skin surface, extracted from skin, permeation at $48 \mathrm{~h}$ and total recovery for PG:PGML, glycerol:PGML and PEG 200:PGML solutions. Actual amounts of active recovered by extraction and measured in receptor compartment also inset $(4 \leq \mathrm{n} \leq 5$; mean $\pm \mathrm{SD})$

The PG:PGML solution delivered $74.24 \%$ of HEX H into the skin. This value was significantly higher than the percentage of HEX $\mathrm{H}$ found on the skin surface (one-way ANOVA, $p<0.001$ ) and also not statistically different from the total recovery (one-way ANOVA, $p>0.05$ ) which, in turn, was significantly higher than the skin surface recovery value (one-way ANOVA, $p<0.001$ ). Contrasting results were observed for the glycerol:PGML and the PEG 200:PGMLsolutions. Their skin extraction recovery values were significantly lower than the corresponding skin surface and total recovery values (one-way ANOVA, $p<0.001$ ). Lastly, the 
percentages of HEX $\mathrm{H}$ recovered on the skin surface for the glycerol:PGML and the PEG 200:PGML solutions were statistically lower than the corresponding total recoveries (one-way ANOVA, $p<0.05$ and $p<0.01$, respectively). The glycerol:PGML and PEG 200:PGML solutions delivered similar amounts of HEX H into the skin $(20.5 \pm 8.2 \mu \mathrm{g}$ and $27.4 \pm 12.4 \mu \mathrm{g}$, respectively) with no significant differences between these values (one-way ANOVA, $p>0.05$ ). The PG:PGML system, however, clearly impacted favourably on topical delivery of HEX $\mathrm{H}$, with significantly higher amounts delivered to skin $(181.0 \pm 35.3 \mu \mathrm{g})$ compared with the other two vehicles (one-way ANOVA, $p<0.001$ ). Total recovery of HEX H was within the accepted range for the PG:PGML vehicle but below the lower limit for the PEG 200:PGML and glycerol:PGML systems. HEX H was previously found to be stable in PBS and PEG 200 (Parisi et al., 2015) but no stability studies were performed in binary mixtures of PEG 200 with PGML or glycerol with PGML. Therefore values for the total recovery of HEX H may reflect stability issues in these solvent combinations and/or skin metabolism as already hypothesised for HEX D.

While HEX $\mathrm{H}$ was used at a concentration of $0.1 \% \mathrm{w} / \mathrm{v}$, saturated solutions of HEX D were prepared in all three PGML systems studied. As a result, HEX D is at its maximal thermodynamic activity and higher skin penetration might be expected for these solutions. Surprisingly, no statistical differences were found between the amounts of HEX D extracted from the skin for glycerol:PGML and PEG 200:PGML and the corresponding amounts of HEX $\mathrm{H}$ for the same vehicles (independentsamples t-test, $p>0.05)$. Moreover, the topical delivery of HEX H from PG:PGML was significantly higher than the combined amounts for HEX D in the skin and in the receptor compartment for the corresponding saturated system (independent-samples t-test, $p<0.001)$., In contrast to HEX D, no HEX H was delivered across the skin from the PG:PGML solution and the active is thus truly targeted to the skin. Overall, it may be concluded that the use of the dihydrochloride salt of hexamidine versus the diisethionate form positively impacted the topical delivery of the active from the PGML binary solvent systems.

The inclusion of PGML in the binary systems with PG, glycerol and PEG 200 was clearly effective in enhancing the topical delivery of both HEX D and HEX H when compared with the neat solvents. The amounts of HEX D extracted from the skin following the application of saturated solutions in PG:PGML, glycerol:PGML and PEG 200:PGML were 17, 15 and 9 times higher than those obtained from the 
corresponding solutions of HEX D in PG, glycerol and PEG 200 (independentsamples t-test, $p<0.01)$. A 50-, 9- and 6-fold increase in the topical delivery of HEX H was obtained for the solutions in PG:PGML, glycerol:PGML and PEG 200:PGML in comparison to the single solvent systems (independent-samples t-test, $p<0.001, p$ $<0.05$ and $p<0.05$, respectively).

PGML is the monoester of lauric acid with PG and is used in dermal and topical drug delivery as a water-in-oil surfactant, emulsion stabiliser and active ingredient solubiliser and is associated with enhanced penetration of actives (Gattefossé, 2010a; Gattefossé, 2010b). Neat PGML, incorporated into a silicone based pressure sensitive adhesive, enhanced estradiol permeation through human cadaver epidermis (Irion et al., 1995). PGML has also been combined with other vehicles and synergistic actions have been demonstrated. Roy et al. (1994), for instance, showed that the addition of 10\% PGML to PG increased buprenorphine flux through human cadaver epidermis by a factor of 7.5 compared with PG alone. In the same study it was also demonstrated that the concomitant inclusion of $15 \%$ PGML and 5\% water to PG led to a 110-fold increase in the transdermal transport of the hydrochloride salt of buprenorphine. The recent work of Mohammed et al. (2014) highlighted the exceptional effect of PG:PGML (50:50) on the delivery of niacinamide across human skin both in-vitro and in-vivo. In the in-vitro permeation studies, the niacinamide flux from the binary solvent system was 437 times higher than that from neat PG. The in-vivo confocal Raman spectroscopy studies showed that the signal intensity of the stratum corneum depth profile of niacinamide was considerably stronger for the PG:PGML system rather than for PG alone. The grade of PGML used in the present work is reported to contain less than $6 \%$ of free saturated fatty acids, the majority being lauric acid (Gattefossé, 2010b). Saturated fatty acids are well known chemical penetration enhancers and their maximal activity is associated with chain lengths of $\mathrm{C}_{9}-\mathrm{C}_{12}$ (Aungst et al., 1986; Aungst, 1989). A number of publications demonstrated both the efficacy of lauric acid and the impact of its synergy with PG on the transdermal deliveries of actives with disparate physicochemical properties using human skin (Green et al., 1988; Roy et al., 1994). Lauric acid has been shown to form ion pairs with cationic actives, thus increasing their hydrophobic nature and enhancing their transport through lipophilic membranes (Green and Hadgraft, 1987; Green et al., 1988; Stott et al., 2001). The presence of lauric acid may therefore have contributed to the enhancing effect of PGML on the 
topical delivery of HEX D and HEX H. It is also notable that most of the literature has focused on the ability of PGML to enhance transdermal rather than topical delivery of actives.

\section{Conclusions}

As we have previously reported, the physicochemical properties of HEX $\mathrm{H}$ suggest that this salt is a better candidate for topical delivery than HEX D. Despite a higher melting point, $\mathrm{HEX} \mathrm{H}$ has a smaller $\mathrm{MW}$ and a higher $\log \mathrm{D}_{\mathrm{o} / \mathrm{w}}$ at $\mathrm{pH}=7.4$ than HEX D. It was thus expected that the various solvent systems evaluated should target HEX $\mathrm{H}$ to skin more effectively than HEX D. Overall the use of the dihydrochloride rather the diisethionate salt did not have a significant impact on the topical delivery of hexamidine. One possible reason for this may be that the increased lipophilicity obtained by the substitution of the isethionate anion with the hydrochloride was not sufficient to produce a marked improvement in penetration of the active through the skin barrier.

Only 10 of the 30 binary solvent systems delivered higher amounts of active into the skin than the corresponding single solvent systems. Interestingly, except for the PG:1,2-PENT solution of HEX D, none of these ten systems contained TC, DMI or 1,2-PENT. DMI did not promote the skin penetration of HEX D and HEX H and its binary systems with PG and glycerol were the only vehicles which did not deliver any HEX D inside the skin. The combination of 1,2-PENT with PG, glycerol and PEG 200 did not improve the topical delivery of HEX D and HEX H with the exception of the solution of HEX D in PG:1,2-PENT. For these particular binary systems it is reasonable to speculate that their poor performance reflected the favourable solubility of both HEX D and HEX H in 1,2-PENT $(15.31 \mathrm{mg} / \mathrm{mL}$ and $22.65 \mathrm{mg} / \mathrm{mL}$, respectively). As a result, the thermodynamic activity of HEX D and HEX $\mathrm{H}$ in binary solvent systems containing 1,2-PENT is comparatively low thus affecting the driving force of the systems and, ultimately, reducing the partitioning of the actives into the stratum corneum. IPA is present in 3 of the 10 binary solvent systems which enhanced topical delivery compared with neat solvents; interestingly, while IPA promoted skin uptake of HEX D, no effect was observed for HEX H.

The 6 binary solvent systems containing PGML in combination with PG, glycerol and PEG 200 complete the set of the ten systems which enhanced the topical delivery of HEX D and HEX H with the PG:PGML system clearly outranking the 
other vehicles. As noted, neat PGML is reported to enhance the percutaneous penetration of several active ingredients and synergistic effects have been reported when PGML is combined with hydrophilic enhancers containing hydroxy groups such as PG. Glycerol, which has three hydroxy groups in its chemical structure, and PEG 200, which has two hydroxy groups and numerous ether groups, are hydrophilic solvents. It may thus be hypothesised that as for PG, the combination of glycerol and PEG 200 with PGML may exert a synergistic action on the skin delivery of active ingredients and, in this case, may have contributed to the enhancement of the topical delivery of HEX D and HEX $\mathrm{H}$. The PGML grade used for these investigations contained small percentages of lauric acid which has enhancing effects in its own right and it also acts synergistically with PG. To determine whether an ion-pair mechanism is relevant for this work further experiments are ongoing.

Finally, it is important to note that the mechanisms of action of the enhancers examined in this study remain to be fully elucidated. Even though we have conducted a comprehensive evaluation of two salts of the same active it is clearly not possible to predict that enhancement effects will be achieved depending on the CPEs selected. We believe this further underlines the necessity for fundamental investigations into the fate of excipients and CPEs when applied to the skin. These studies will provide important insights for rational formulation design in the future.

Acknowledgements: NP is very grateful for financial support from Procter \& Gamble, UK. 


\section{References}

Aungst, B.J., Rogers, N.J. and Shefter, E., 1986. Enhancement of naloxone penetration through human skin in vitro using fatty acids, fatty alcohols, surfactants, sulfoxides and amides. Int. J. Pharm. 33(1-3):225-234.

Aungst, B.J., 1989. Structure/effect studies of fatty acid isomers as skin penetration enhancers and skin irritants. Pharm. Res. 6(3):244-247.

Bennett, S.L., Barry, B.W. and Woodford, R., 1985. Optimization of bioavailability of topical steroids: non-occluded penetration enhancers under thermodynamic control. J. Pharm. Pharmacol. 37 (5):298-304.

Caon T, Costa AC, de Oliveira MA, Micke GA, Simões CM. 2010. Evaluation of the transdermal permeation of different paraben combinations through a pig ear skin model. Int. J. Pharm. 391(1-2):1-6.

Cosmetic Ingredient Review Expert Panel, 2007. Final report on the safety assessment of hexamidine and hexamidine diisethionate. Int. J. Toxicol. 26(Suppl. 3):79-88.

Duracher, L., Blasco, L., Hubaud, J.C., Vian, L. and Marti-Mestres, G., 2009. The influence of alcohol, propylene glycol and 1,2-pentanediol on the permeability of hydrophilic model drug through excised pig skin. Int. J. Pharm. 374(1-2):39-45.

Franz, T.J. 1975. Percutaneous absorption on the relevance of in vitro data. J. Invest. Dermatol. 64(3):190-5.

Funke, A.P., Schiller, R., Motzkus, H.W., Günther, C., Müller, R.H. and Lipp, R., 2002. Transdermal delivery of highly lipophilic drugs: in vitro fluxes of antiestrogens, permeation enhancers, and solvents from liquid formulations. Pharm. Res. 19(5):661-668.

Gattefossé, 2010a. Lauroglycol ${ }^{\mathrm{TM}} 90$ - Propylene glycol monolaurate (type II) EP/NF. [online]. Available at: <http://www.gattefosse.com/node.php?articleid=164?> [Accessed 17 August 2015].

Gattefossé, 2010b. Technical data sheet - Lauroglycol ${ }^{\mathrm{TM}}$ 90. Gattefossé Document Centre [online]. Available at: $\langle$ http://www.gattefosse.com/media/document/tds_lauroglycol_90.PDF> [Accessed 01 July 2015].

Green, P.G. and Hadgraft, J., 1987. Facilitated transfer of cationic drugs across a lipoidal membrane by oleic acid and lauric acid. Int. J. Pharm. 37(3):251-255.

Green, P.G., Guy, R.H. and Hadgraft, J., 1988. In vitro and in vivo enhancement of skin permeation with oleic and lauric acids. Int. J. Pharm. 48(1-3):103-111. 
Hadgraft, J., 1999. Passive enhancement strategies in topical and transdermal drug delivery. Int. J. Pharm. 184(1):1-6.

Hadgraft, J., Lane, M.E. Drug crystallization - implications for topical and transdermal delivery. Expert Opin. Drug Deliv. 2016. DOI: $10.1517 / 17425247.2016 .1140146$.

Harrison, J.E., Watkinson, A.C., Green, D.M., Hadgraft, J. and Brain, K., 1996. The relative effect of Azone ${ }^{\circledR}$ and Transcutol ${ }^{\circledR}$ on permeant diffusivity and solubility in human stratum corneum. Pharm. Res. 13 (4):542-546.

Hirata, K., Helal, F., Hadgraft, J. and Lane, M.E., 2013. Formulation of carbenoxolone for delivery to the skin. Int. J. Pharm., 448(2): 360-365.

Irion, G.D., Garrison, M.D. and Abraham, W., 1995. Effect of PGML excipient mixture in a transdermal system on the in vitro transport of estradiol across skin. Pharm. Res. 12(11):1618-1622.

Jarrold, B.B., Tiesman, J., Robinson, M., Binder, R. and Osborne, R., 2010a. HEX, a protease inhibitor, promotes stratum corneum lipid biomarkers in vitro. J. Am. Acad. Dermatol. 62(3, Suppl. 1):AB2.

Jarrold, B.B., Kaczvinsky, J., Matts, P.J. and Osborne, R., 2010b. Use of a cosmetic moisturizer promotes corneocyte maturity. J. Am. Acad. Dermatol. 62(3, Suppl. 1) :AB62.

Kimball, A.B., Grant, R.A., Wang, F., Osborne, R. and Tiesman, J.P., 2012. Beyond the blot: cutting edge tools for genomics, proteomics and metabolomics analyses and previous successes. Br. J. Dermatol. 166(Suppl. S2):1-8.

Lane, M.E., 2013. Skin penetration enhancers. Int. J. Pharm. 447(1-2):12-21.

Mohammed, D., Matts, P.J., Hadgraft, J. and Lane, M.E., 2014. In Vitro-In Vivo Correlation in Skin Permeation. Pharm. Res. 31(2):394-400.

Oliveira, G., Hadgraft, J. and Lane, M.E., 2012. The influence of volatile solvents on transport across model membranes and human skin. Int. J. Pharm. 435(1):38-49.

Organisation for Economic Cooperation and Development, 2004. Test No. 428: skin absorption: in vitro method. OECD guidelines for the testing of chemicals, section 4: health effects. Paris: OECD Publishing, pp.1-8.

Organisation for Economic Cooperation and Development, 2011. Guidance notes on dermal absorption. OECD environment, health and safety publications, series on testing and assessment, No. 156. Paris, OECD Publishing, pp. 1-72.

Osborne, R., Mullins, L.A. and Jarrold, B.B., 2009. Understanding metabolic pathways for skin anti-aging. J. Drugs Dermatol. 8 (Suppl. 7):s4-s7. 
Osborne, D.W., 2011. Diethylene glycol monoethyl ether: an emerging solvent in topical dermatology products. J. Cosmetic Dermatol. 10 (4):324-329.

Parisi, N., Matts, P.J., Lever, R., Hadgraft, J., Lane M.E. 2015. Preparation and characterisation of hexamidine salts. Int. J. Pharm., 493(1-2):404-11.

Puglia, C. and Bonina, F., 2008. Effect of polyunsaturated fatty acids and some conventional penetration enhancers on transdermal delivery of atenolol. Drug Delivery. 15 (2):107-112.

Robin, J. Contact dermatitis to hexamidine. 1978. Contact Dermatitis. 4(6):375-6.

Roy, S.D., Roos, E. and Sharma, K., 1994. Transdermal delivery of buprenorphine through cadaver skin. J. Pharm. Sci. 83 (2):126-130.

Stott, P.W., Williams, A.C. and Barry, B.W., 2001. Mechanistic study into the enhanced transdermal permeation of a model $\beta$-blocker, propranolol, by fatty acids: a melting point depression effect. Int. J. Pharm. 219 (1-2):161-176.

Sullivan Jr., D.W., Gad, S.C. and Julien, M., 2014. A review of the nonclinical safety of Transcutol ${ }^{\circledR}$, a highly purified form of diethylene glycol monoethyl ether (DEGEE) used as a pharmaceutical excipient. Food Chem. Toxicol. 72:40-50.

Tsai, J.C., Cappel, M.J., Flynn, G.L., Weiner, N.D., Kreuter, J. and Ferry, J.J., 1992. Drug and vehicle deposition from topical applications: use of in vitro mass balance technique with minoxidil solutions. J. Pharm. Sci. 81 (8):736-743. 